\title{
Integrated assessment of global water scarcity over the 21st century under multiple climate change mitigation policies
}

M. I. Hejazi et al.

Correspondence to: M. I. Hejazi (mohamad.hejazi@pnnl.gov) 
Figure S1 shows a schematic of the Global Water Availability Model (GWAM) structure

Figure S2 shows a detailed flowchart schematic of the GWAM model calculation algorithm.

Figure S3: Comparison of this study's global runoff volume to other data-based and model-based estimates; the bar denotes the highest and lowest simulated runoff

Figure S4: Comparison of this study's continental runoff volumes to other data-based and modelbased estimates; the bar denotes the highest and lowest simulated runoff

Figure S5 shows the radiative forcing trajectories based on each of the four SRES emission scenarios (A1fi, A2, B2, and B1); blue and red dashed lines are GCAM equivalent simulations of the UCT and FFICT tax regimes to replicate SRES's emission pathways.

Figure S6 shows the the resulting carbon price, $\mathrm{CO} 2$ emission and concentration, and mean global temperature change associated with both the UCT and FFICT tax regimes and all four emission scenarios (A1fi, A2, B2, and B1); the A1fi scenario (black solid line) reflects the noclimate policy scenario (baseline)

Figure S7: Piechart distributions of global water demands (withdrawals) by sector for the baseline scenario (A1fi) in years 2005 \& 2095, and for each of the climate change mitigation policies (2 tax regimes (UCT \& FFICT) x 3 emission scenarios (A2, B2, and B1)

Figure S8 shows the total non-agricultural water demand (e.g., municipal, electricity generation, primary energy, manufacturing water demands) in years 2005, 2050, and 2095 under the baseline scenario. The increases in India and Eastern China are most pronounced

Figure S9 shows the total irrigation water demand in years 2005, 2050, and 2095under the baseline scenario. Regions such as Northern India, Northeastern China, and Southeastern Asia face dramatic increases in irrigation water demand in this scenario.

Figure S10 shows the total livestock water demand in years 2005, 2050, and 2095 under the baseline scenario. With higher population growth and greater demand for food (dairy and meat), places such India, China, Central Africa, and Brazil experience the largest increase in livestock water demand by the end of the $21^{\text {st }}$ century under the baseline scenario. Note, livestock water demand is relatively minor in comparison with the total non-agricultural (Figure S5) and irrigation (Figure S6) water demands.

Figure S11 shows the total agricultural water demand (e.g., irrigation and livestock) in years 2005, 2050, and 2095 under the baseline scenario.

Figure S12 shows the total water demand (including both agricultural and non-agricultural sectors) in years 2005, 2050, and 2095 under the baseline scenario. The greatest increase in water demand is concentrated around Northern India and Eastern China. 
Figure S13 shows the ensemble mean annual runoff in year 2005, 2050, and 2095; annual values are averaged over 2001-2010, 2046-2055, and 2091-2100, respectively; ensemble is based on four GCMs and the A1fi emission scenario. The latter two periods are ensemble mean annual runoff values based on using four GCMs to simulate GWAM in the future.

Figure S14 shows the the water scarcity index (WSI) in years 2005, 2050, and 2095 under the baseline scenario; a value close to one indicates extreme water stress condition while a value close to zero indicates abundant water resources as compared to demands.

Figure S15shows the change in (a) total water demands, (b) total water availability, and (c) water scarcity index between 2095 and 2005 under the baseline scenario.

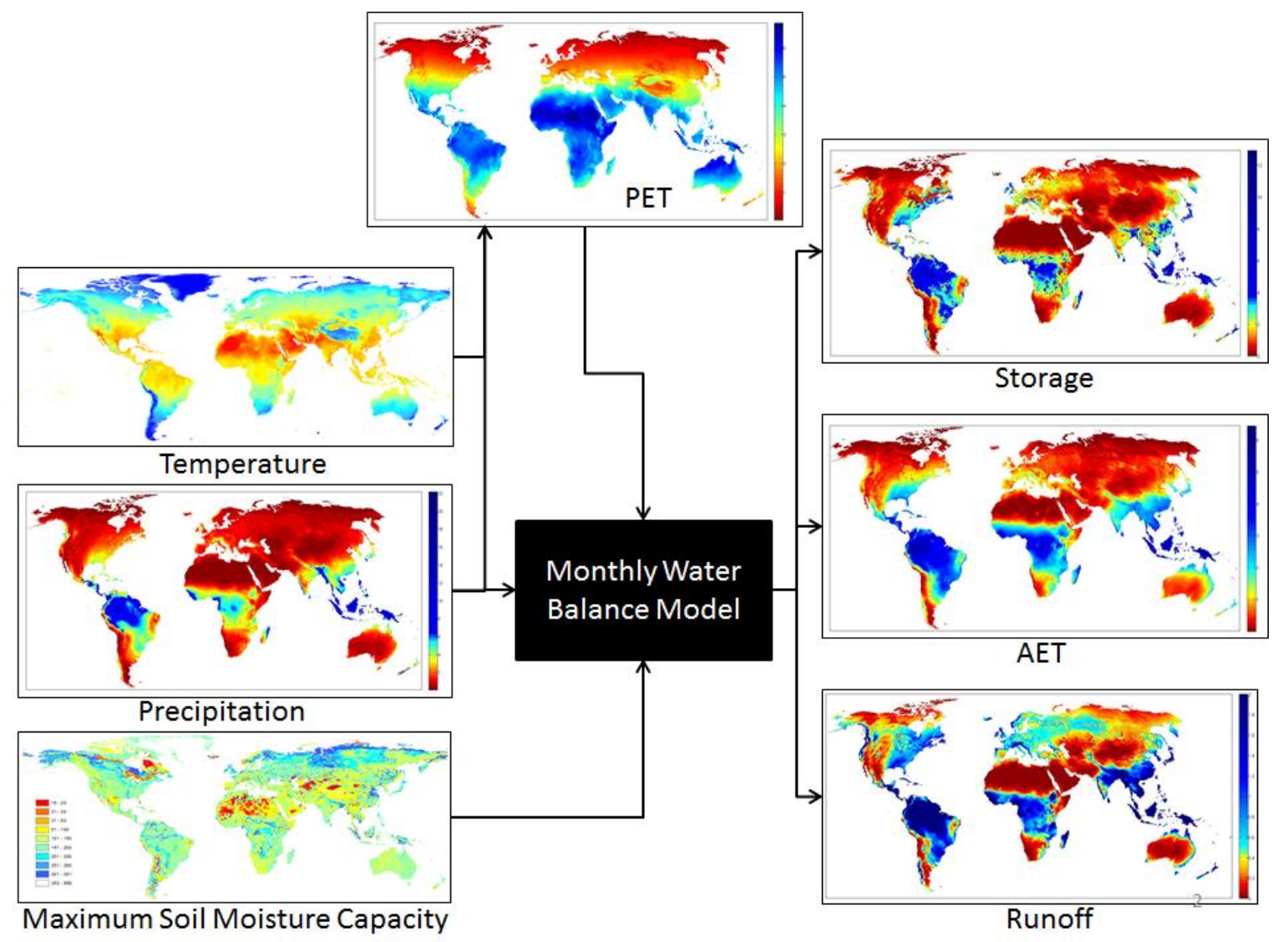

Figure S1: Schematic of the global water availability model (GWAM) structure 


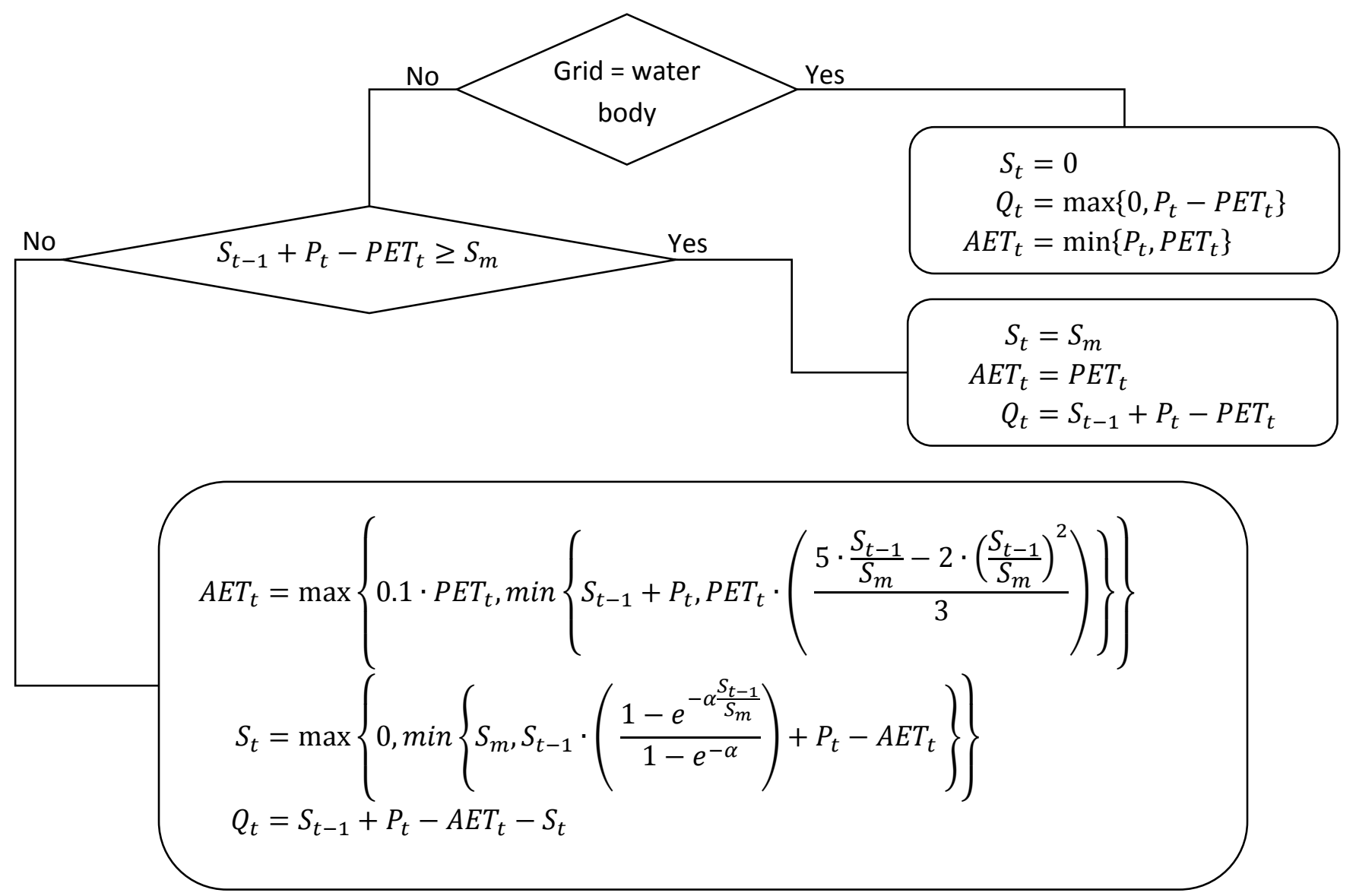

Figure S2: Flowchart diagram of the monthly water-balance modeling algorithm 


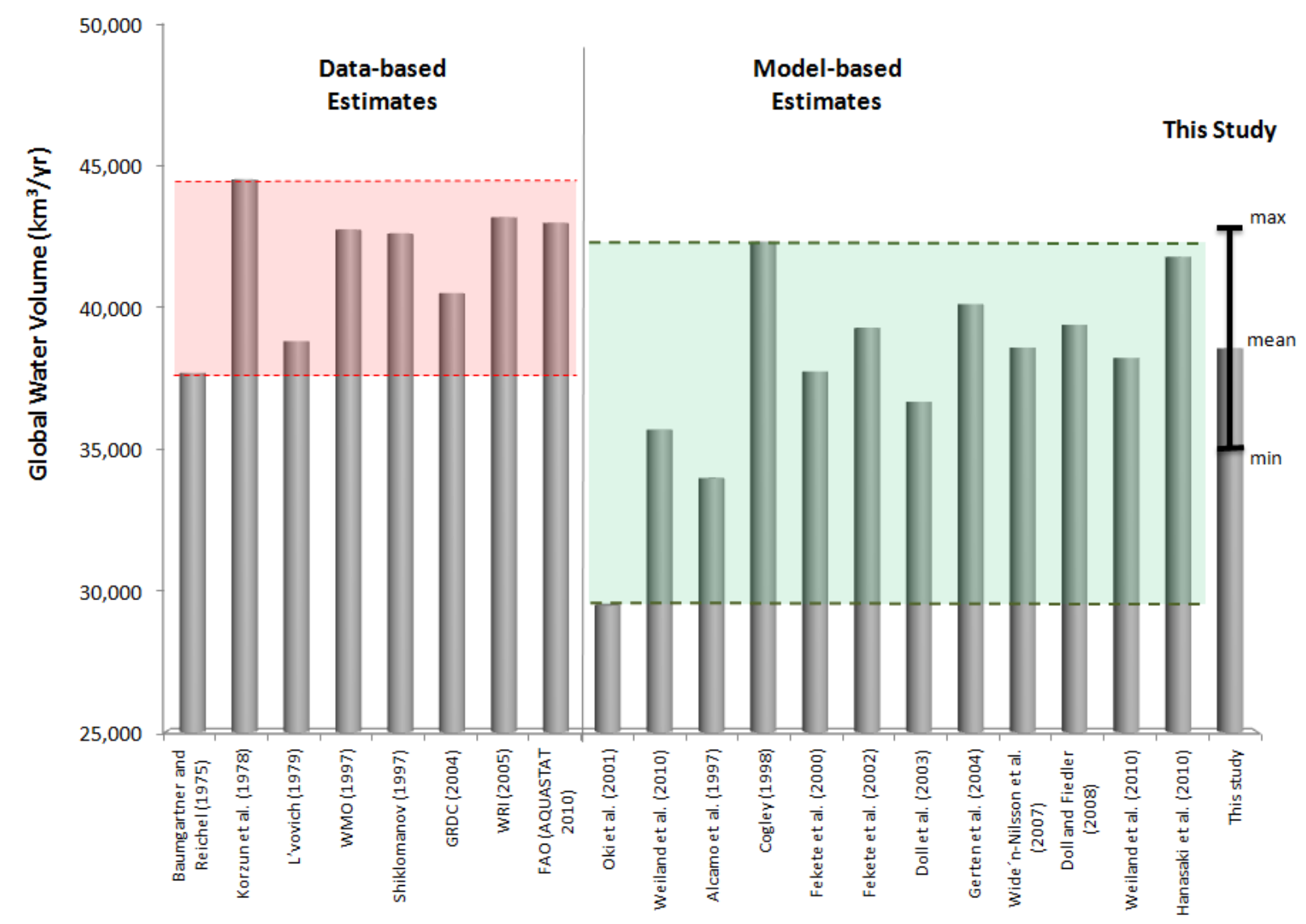

Figure S3: Comparison of this study's global runoff volume to other data-based and model-based estimates; the bar denotes the highest and lowest simulated runoff 


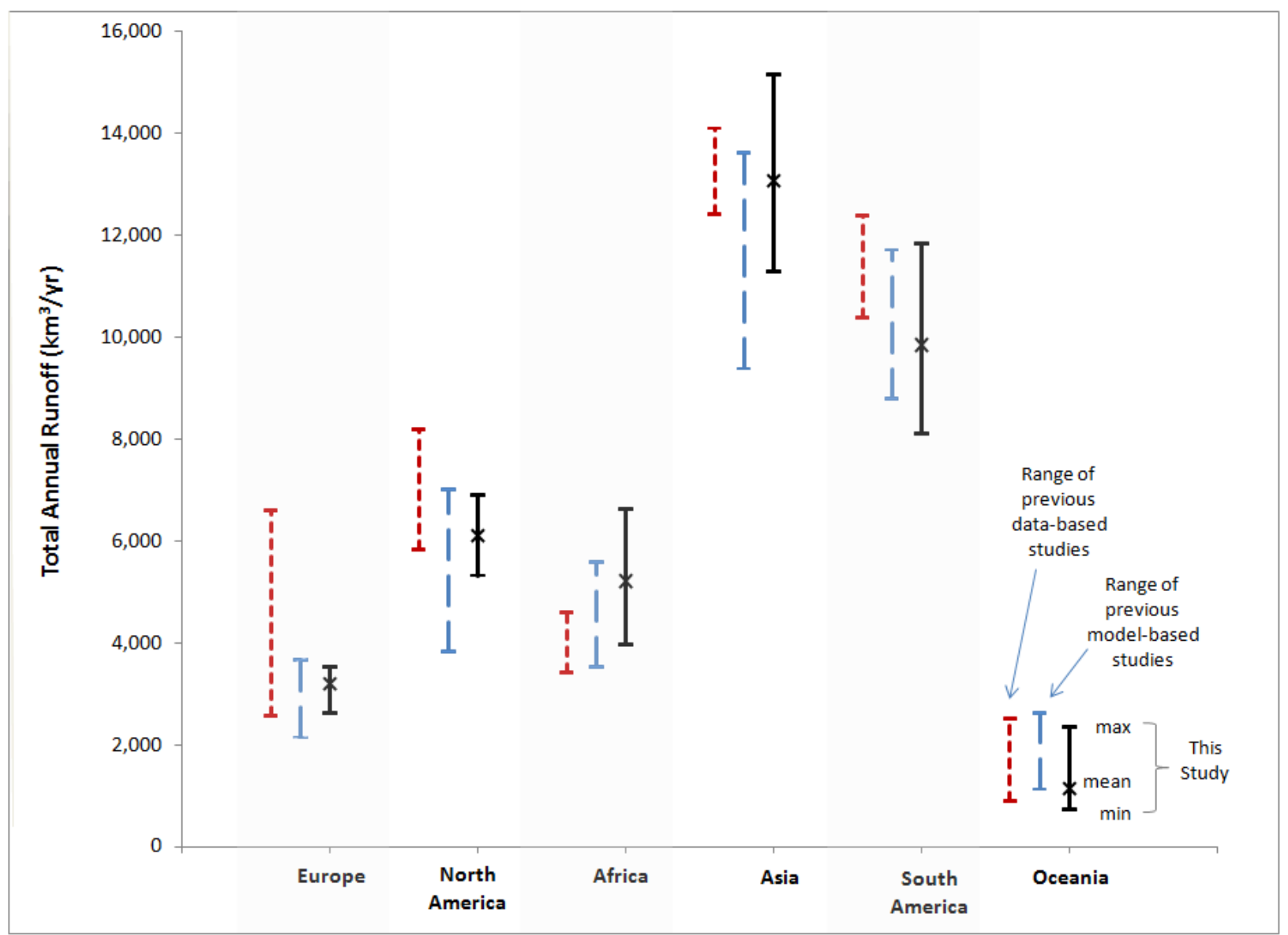

Figure S4: Comparison of this study's continental runoff volumes to other data-based and modelbased estimates; the bar denotes the highest and lowest simulated runoff 

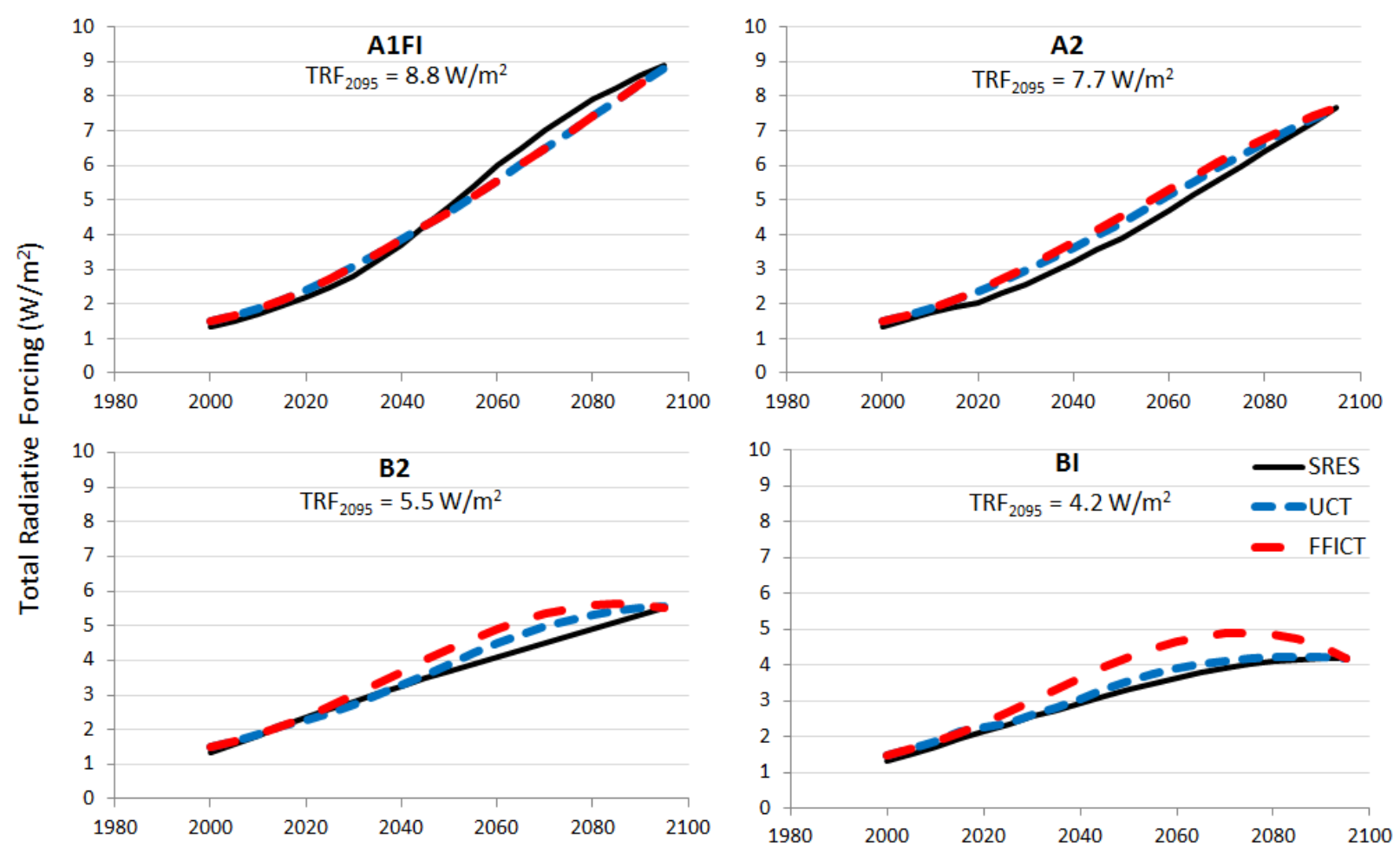

Figure S5: Radiative forcing trajectories based on each of the four SRES emission scenarios (A1fi, A2, B2, and B1); blue and red dashed lines are GCAM equivalent simulations of the UCT and FFICT tax regimes to replicate SRES's emission pathways. 

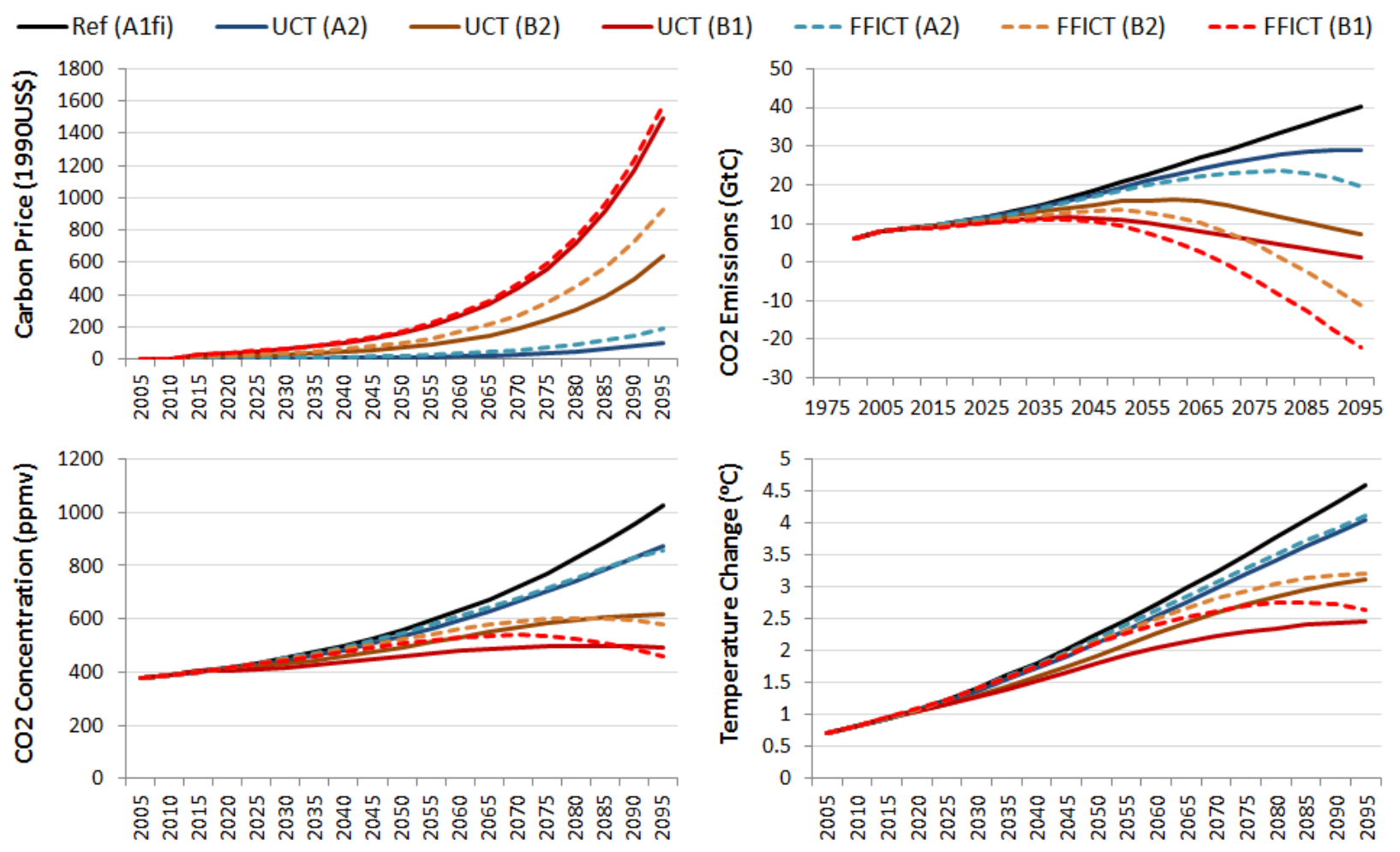

Figure S6: The resulting carbon price, $\mathrm{CO} 2$ emission and concentration, and mean global temperature change associated with both the UCT and FFICT tax regimes and all four emission scenarios (A1fi, A2, B2, and B1); the A1fi scenario (black solid line) reflects the no-climate policy scenario (baseline) 

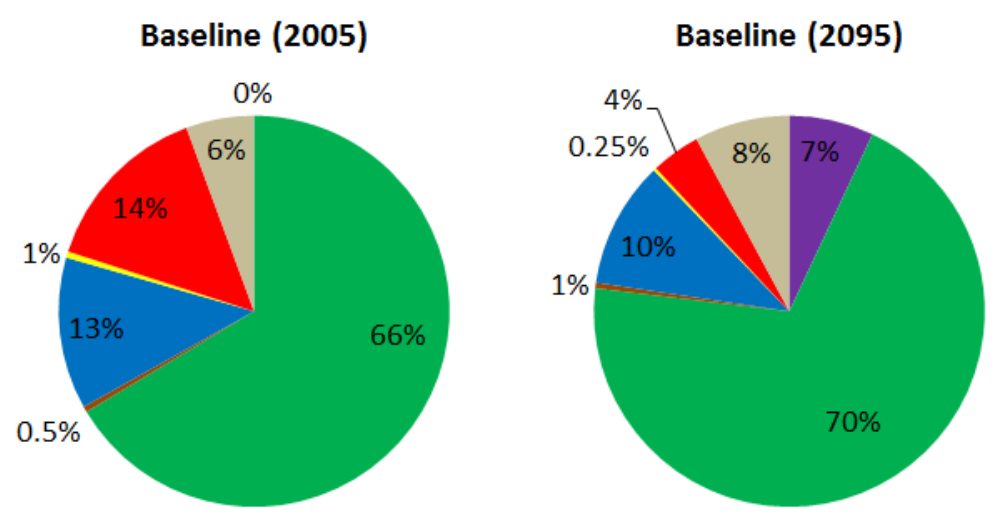

\section{Global Water Withdrawals}

- Biomass

- Crops

- Livestock

Domestic

Primary Energy

- Electricity

Manufacturing

\section{UCT7.7 (2095)}
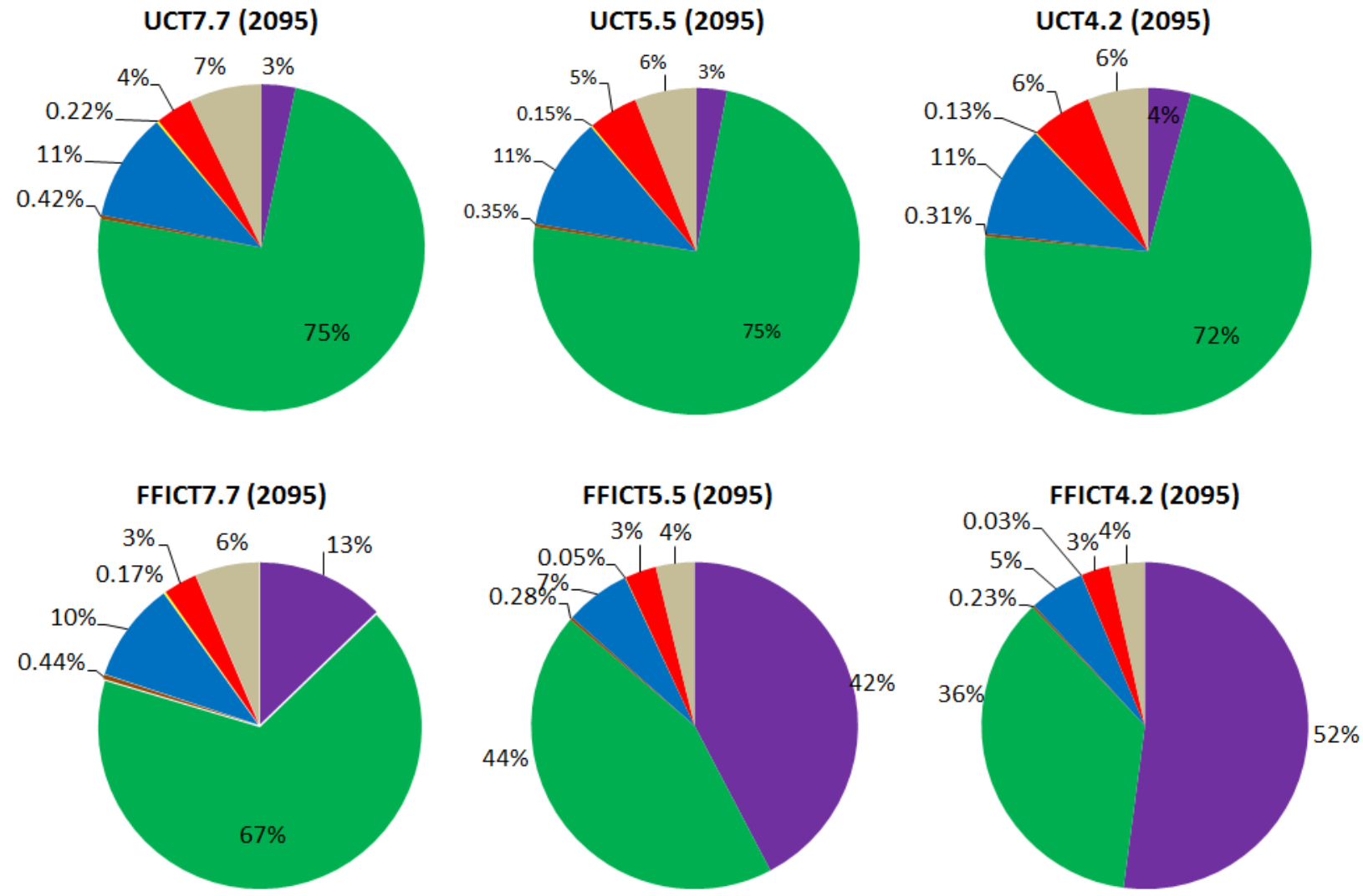

Figure S7: Piechart distributions of global water demands (withdrawals) by sector for the baseline scenario (A1fi) in years 2005 \& 2095, and for each of the climate change mitigation policies ( 2 tax regimes (UCT \& FFICT) $\times 3$ emission scenarios (A2, B2, and B1) 

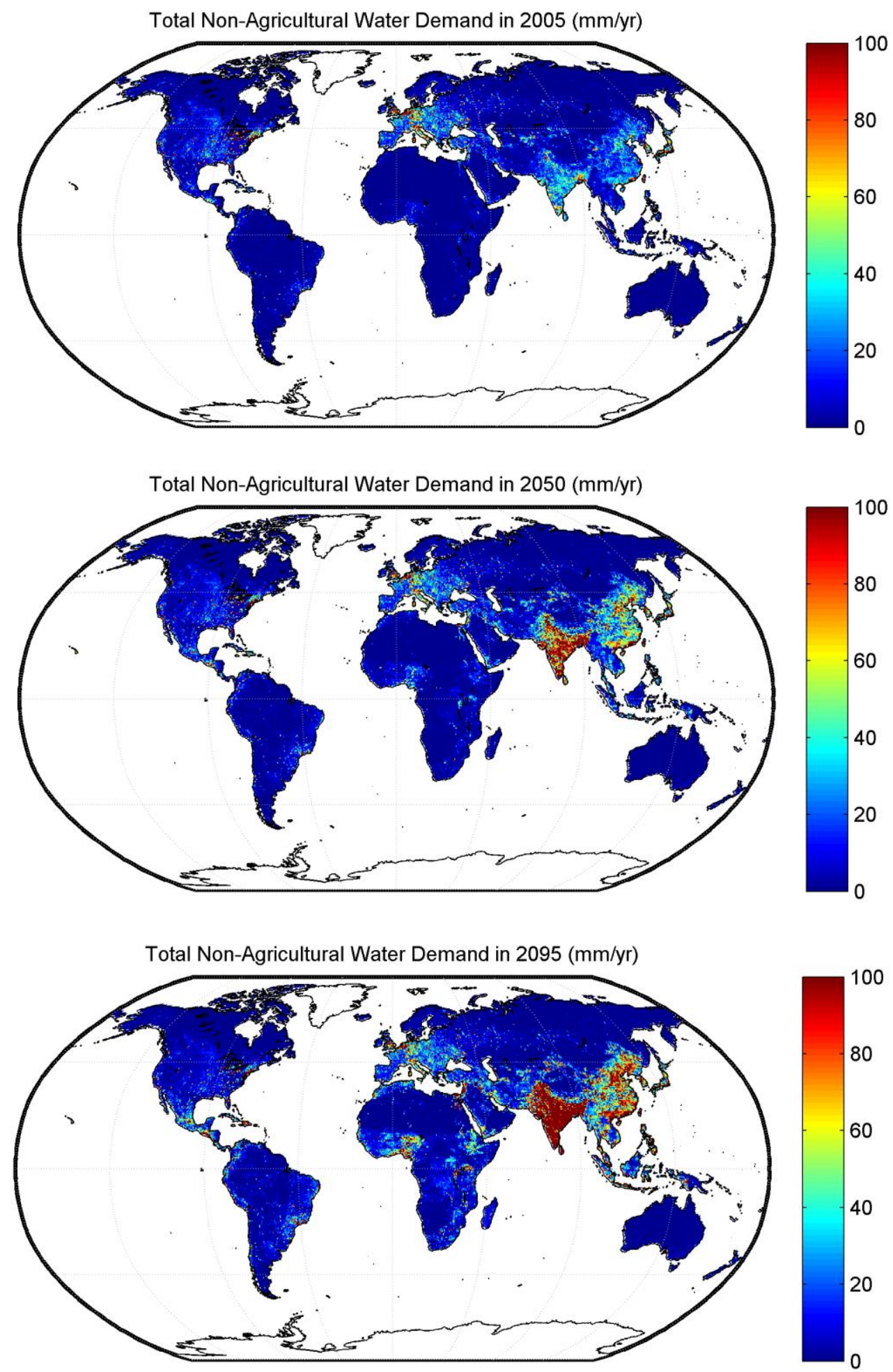

Figure S8: Total non-agricultural water demand (e.g., municipal, electricity generation, primary energy, manufacturing water demands) in years 2005, 2050, and 2095 

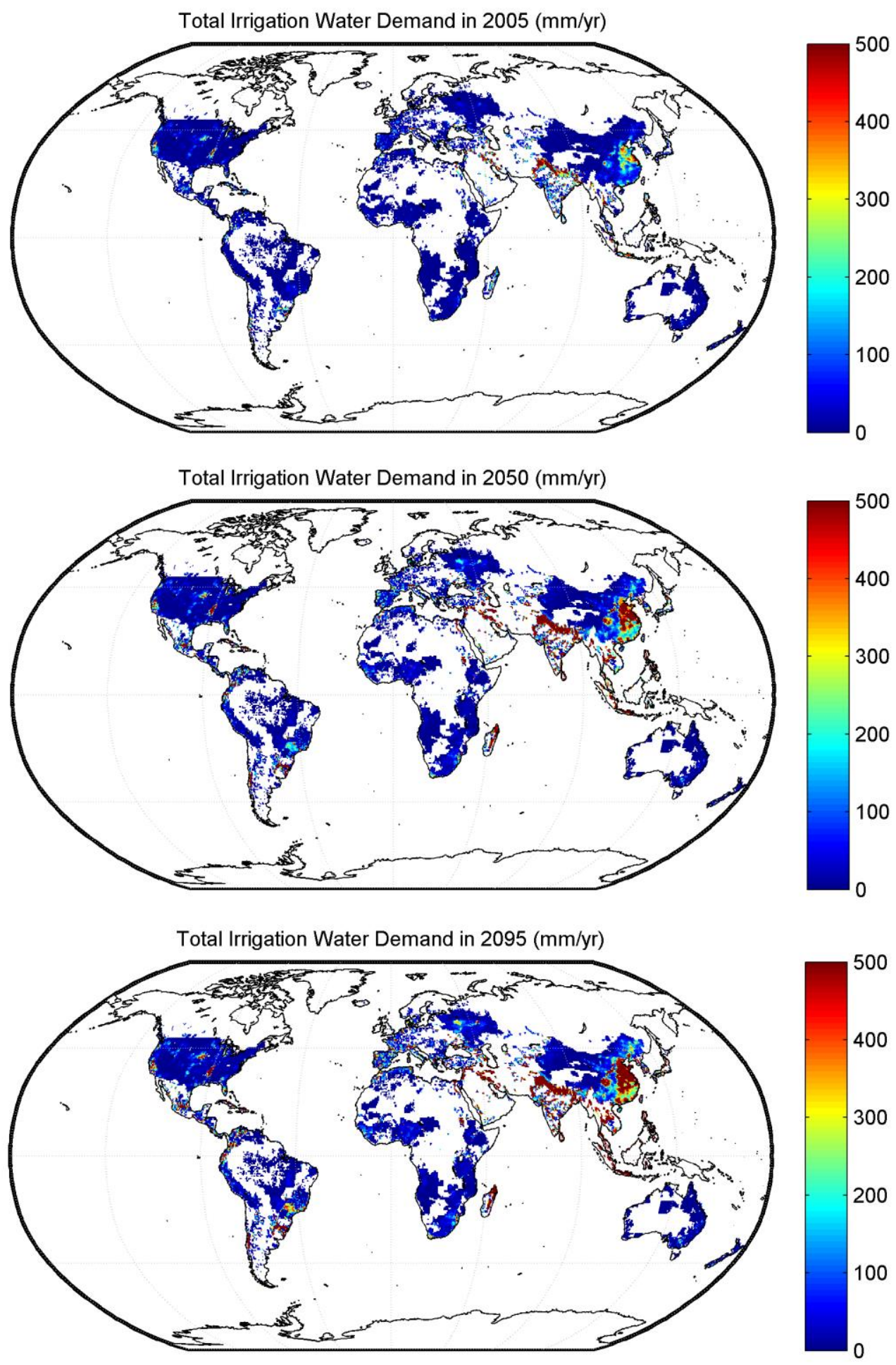

Figure S9: Total irrigation water demand in years 2005, 2050, and 2095 

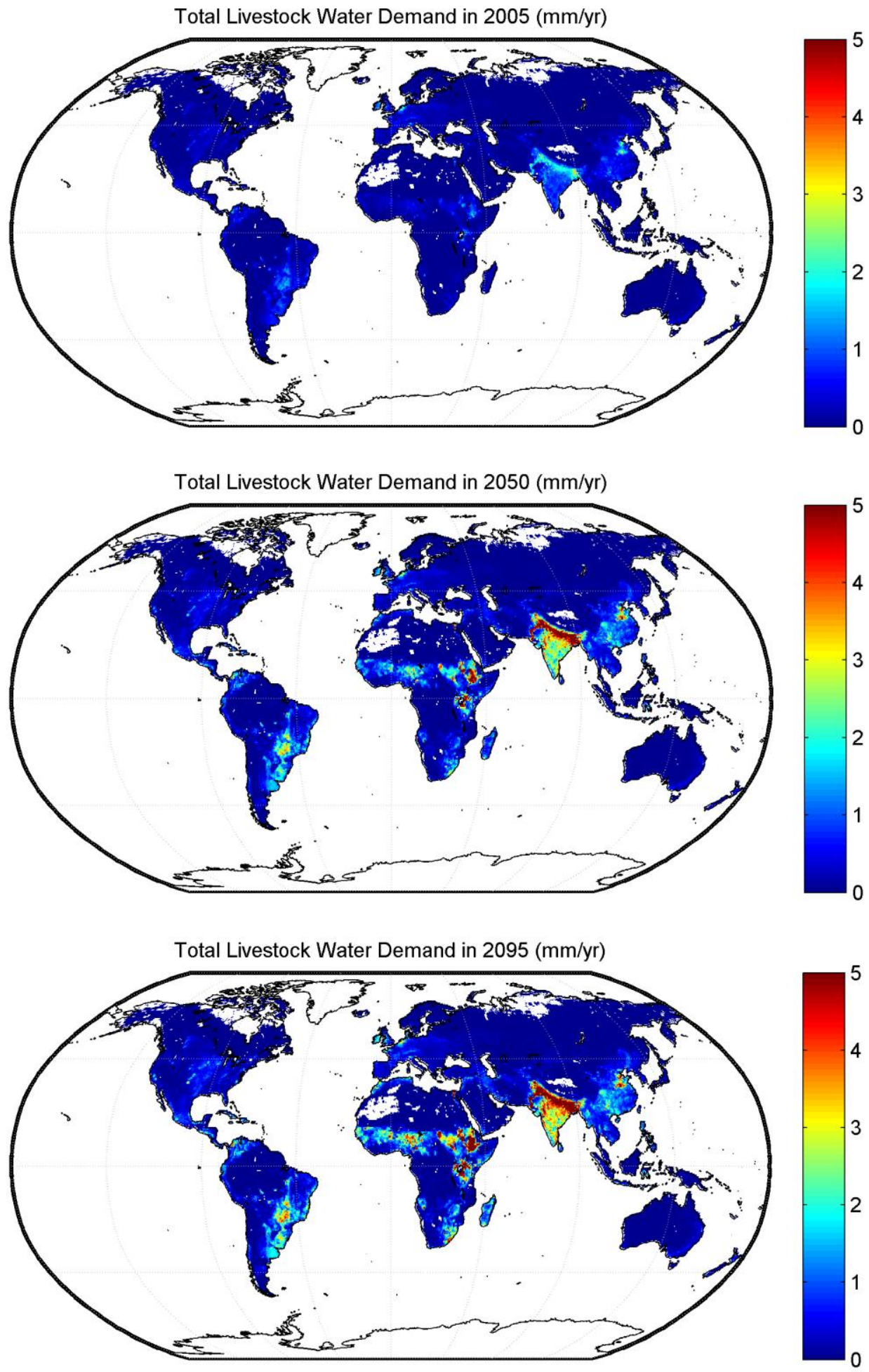

Figure S10: Total livestock water demand in years 2005, 2050, and 2095 

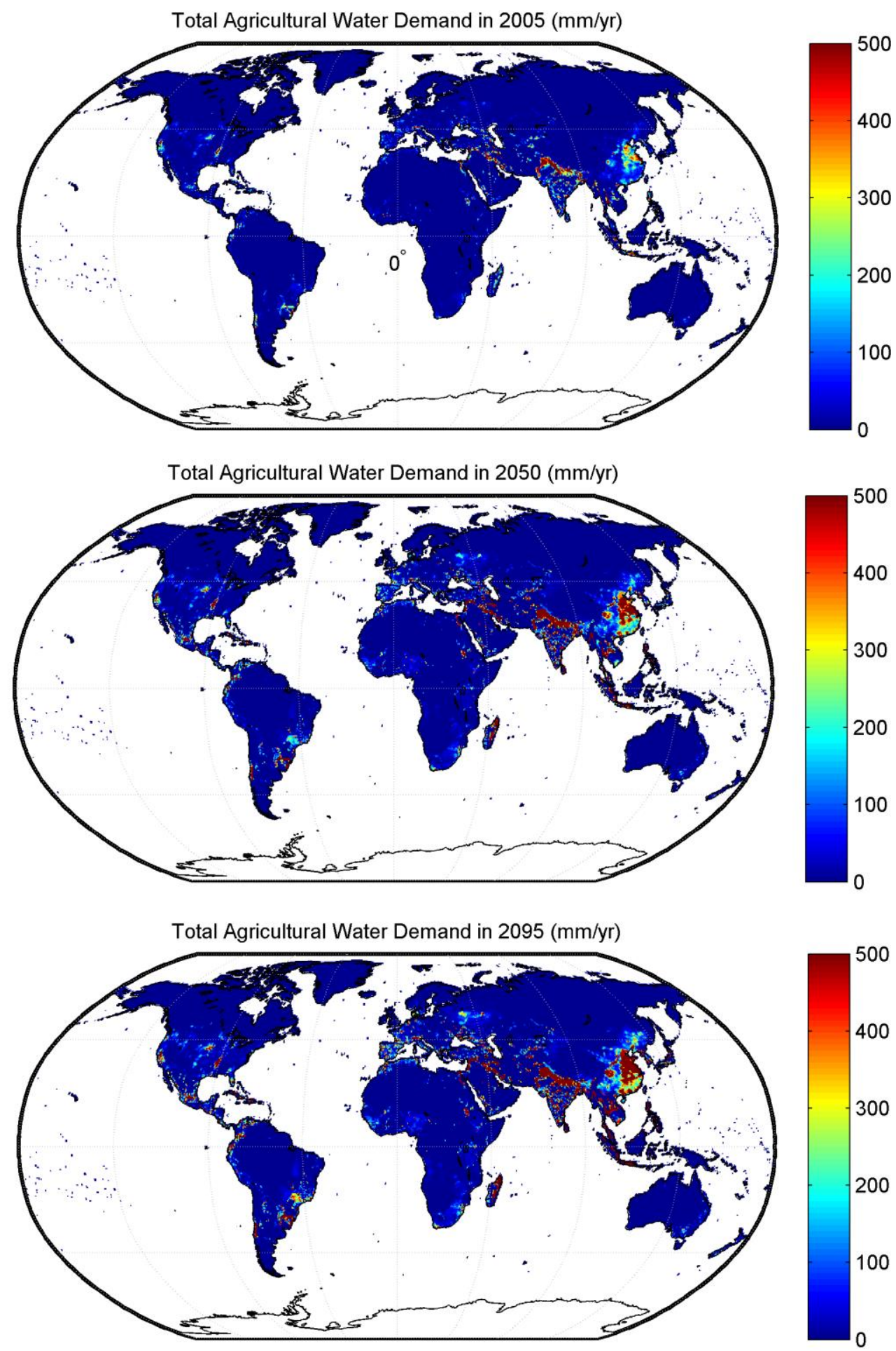

Figure S11: Total agricultural water demand (e.g., irrigation and livestock) in years 2005, 2050, and 2095 

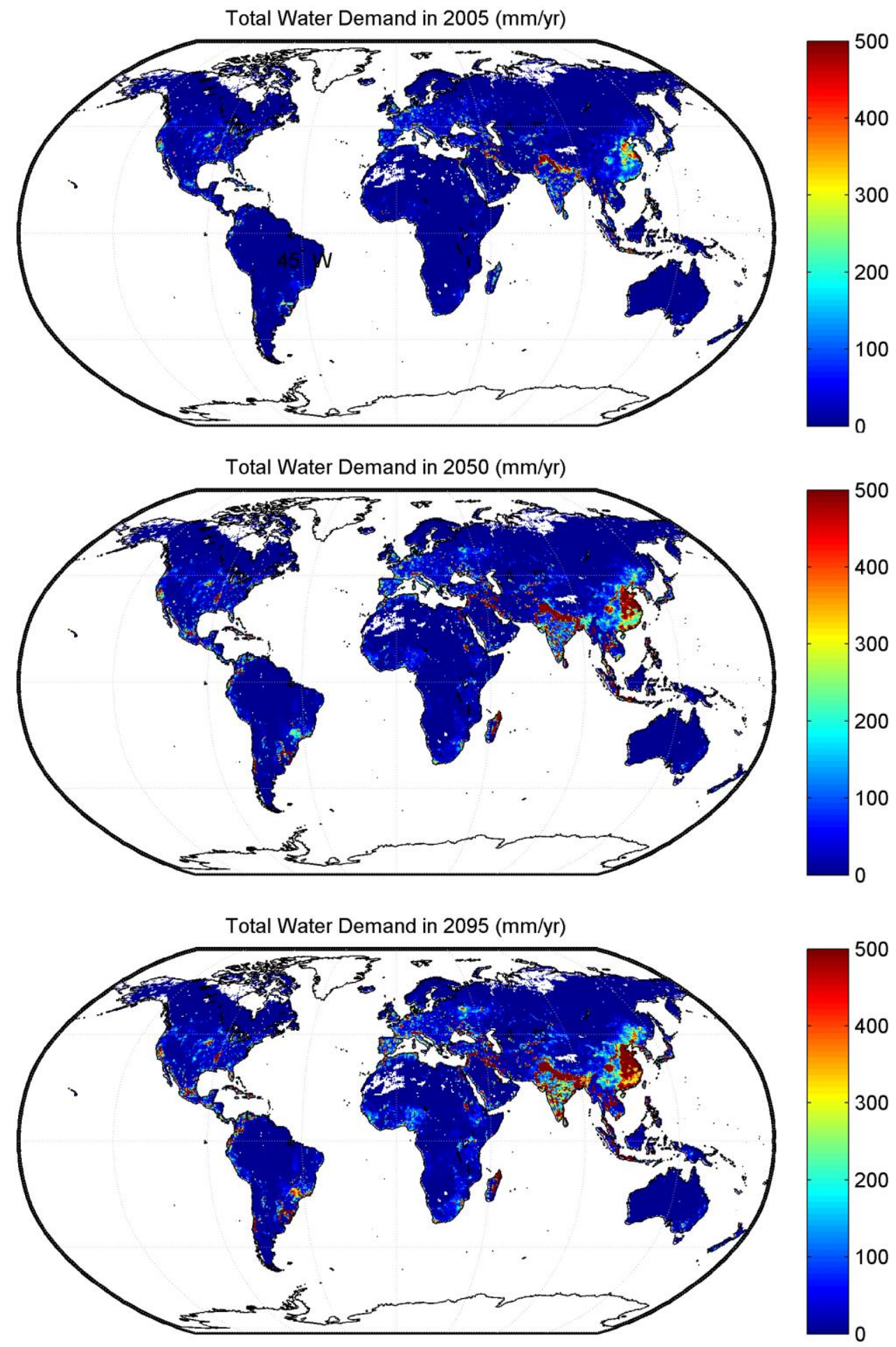

Figure S12: Total water demand (including both agricultural and non-agricultural sectors) in years 2005, 2050, and 2095 

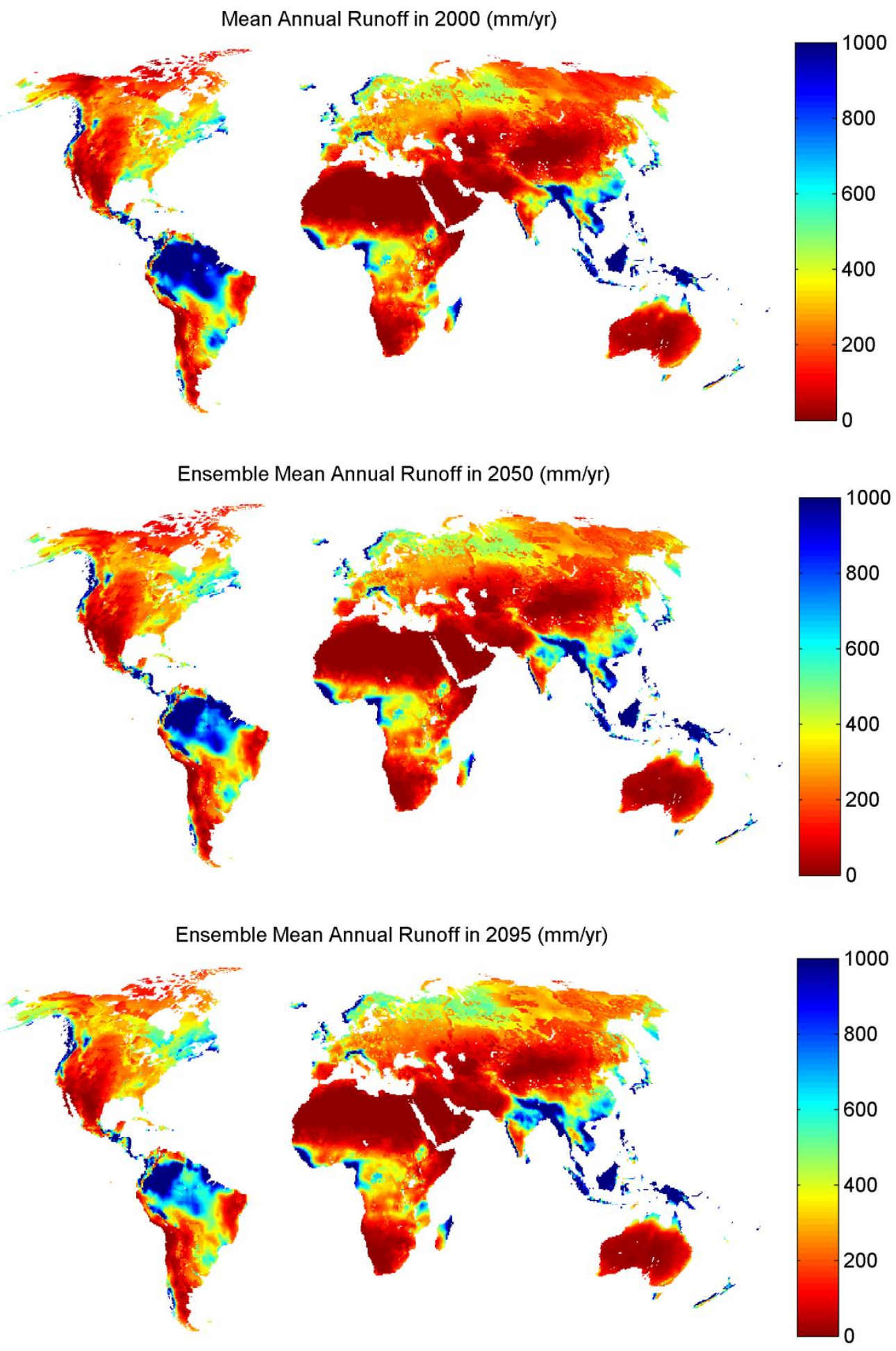

Figure S13: Ensemble mean annual runoff in year 2005, 2050, and 2095; annual values are averaged over 2001-2010, 2046-2055, and 2091-2100, respectively; ensemble is based on four GCMs and the A1fi emission scenario. 

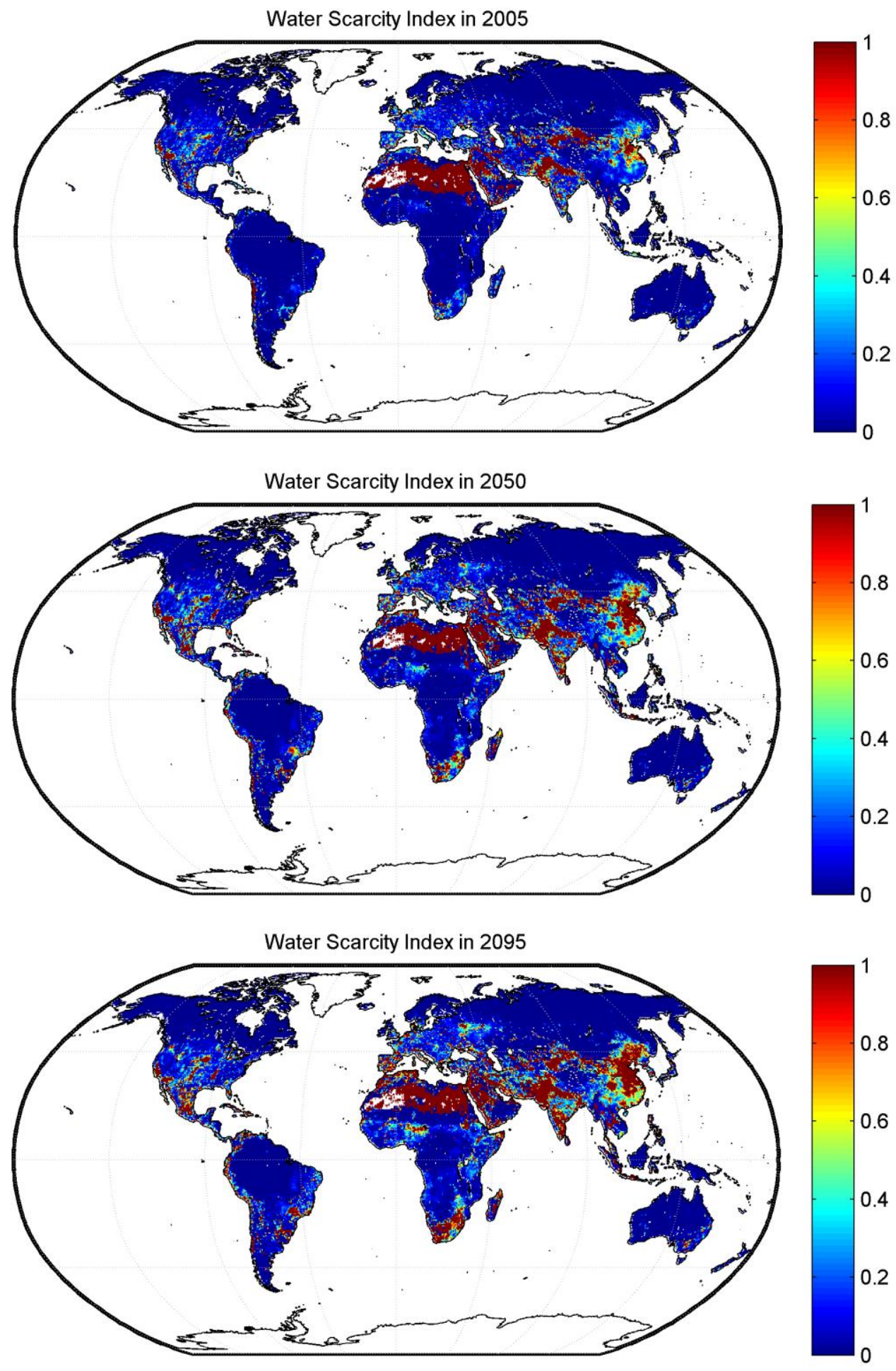

(a) Grid-scale water scarcity 

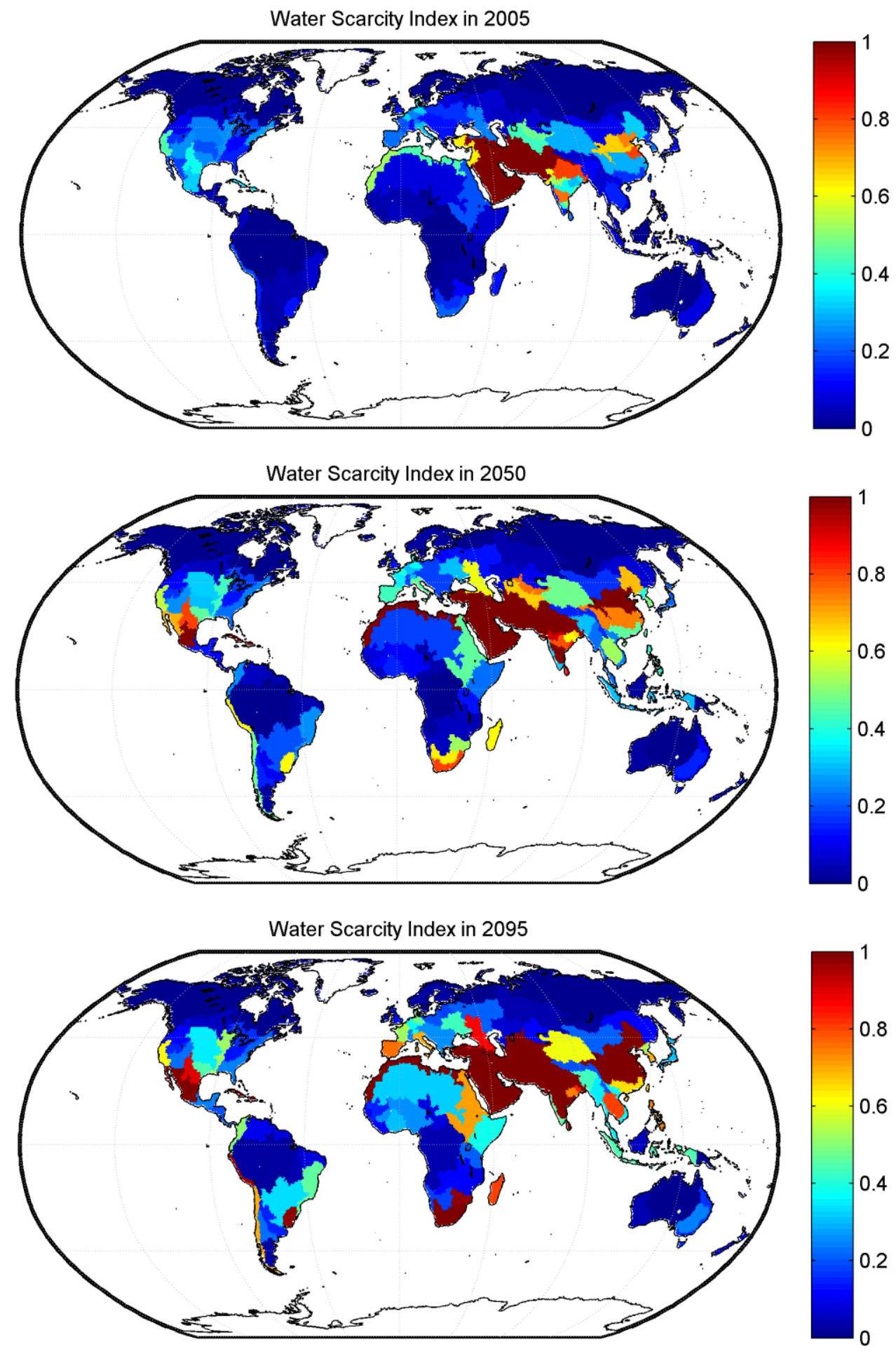

(b) Basin-scale water scarcity

Figure S14: The water scarcity index (WSI) in years 2005, 2050, and 2095; a value close to one indicates extreme water stress condition while a value close to zero indicates abundant water resources as compared to demands. 
(a)

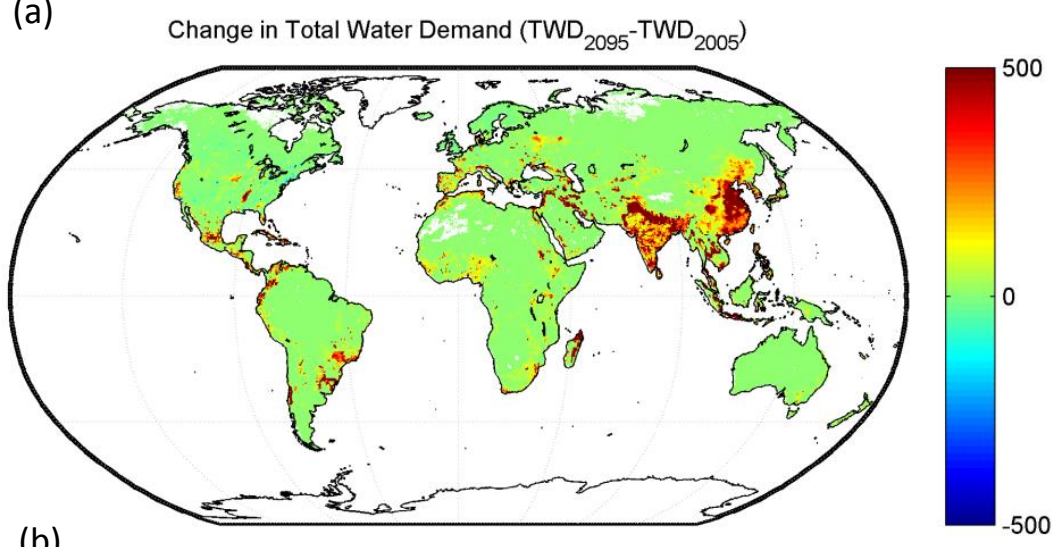

(b)

Change in Ensemble Mean Annual Runoff $\left.\mathbb{Q}_{2095}{ }^{-} Q_{2005}\right)(\mathrm{mm} / \mathrm{yr})$

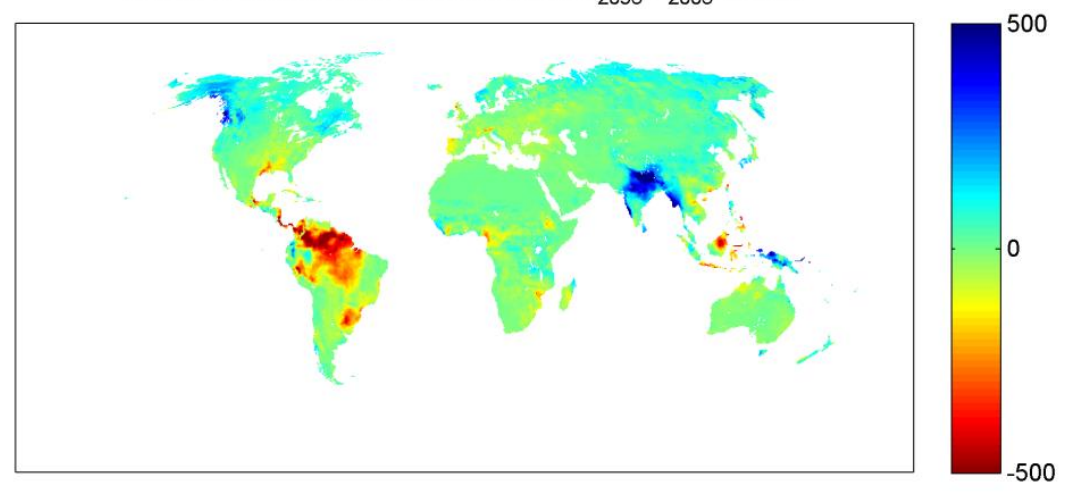

(c)

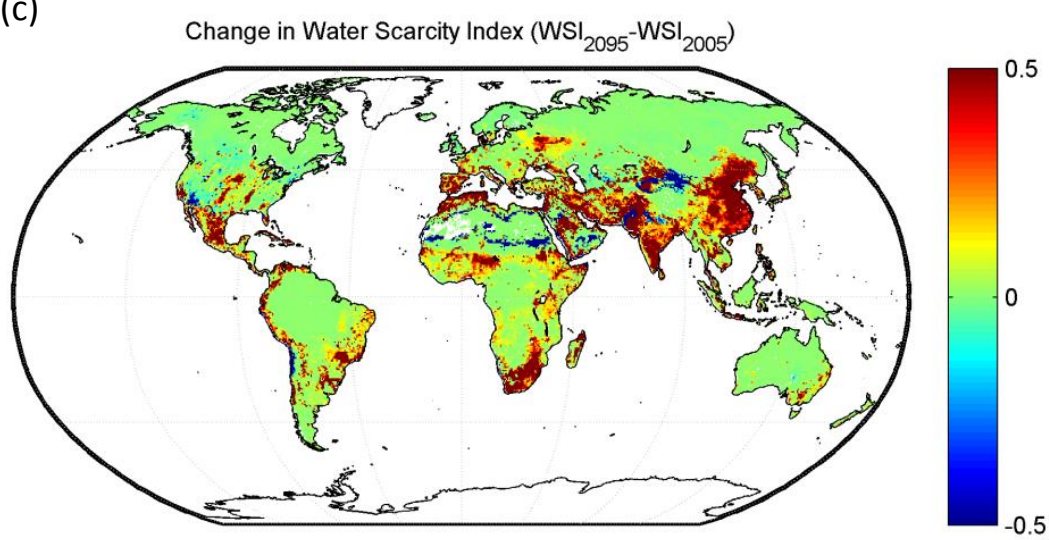

(d)

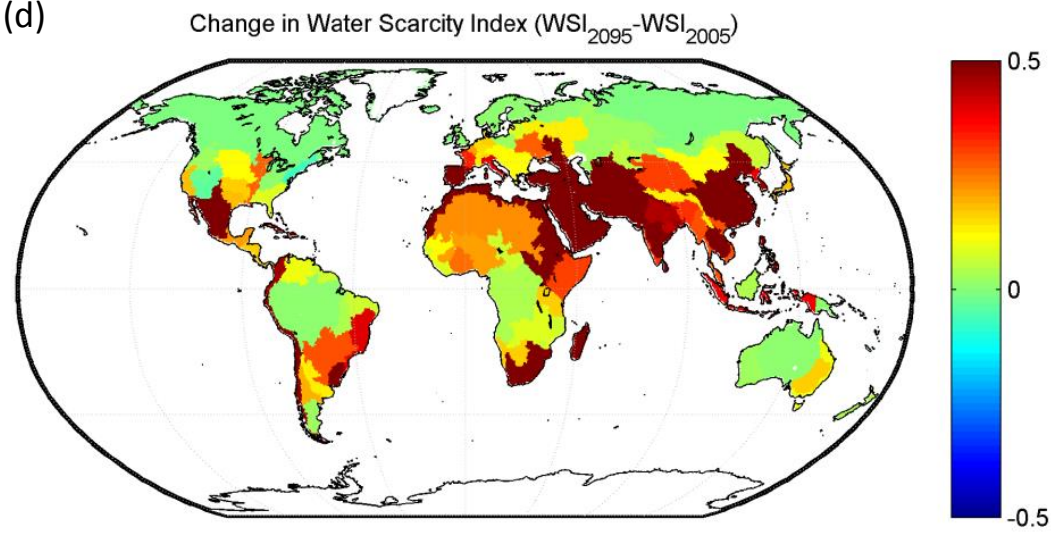

Figure S15: change in (a) total water demands, (b) total water availability, and (c) water scarcity index between 2095 and 2005 
Table S1: Average annual runoff $\left[\mathrm{km}^{3} / \mathrm{yr}\right]$ at the country scale in comparison with other data-based and model based estimates.

\begin{tabular}{|c|c|c|c|c|c|c|c|}
\hline Country Name & $\begin{array}{r}\text { AQUASTAT } \\
\text { FAO* } \\
(2010) \\
\end{array}$ & $\begin{array}{r}\text { WRI } \\
(2005) \\
\end{array}$ & $\begin{array}{r}\text { Shiklomanov } \\
(2000) \\
\end{array}$ & $\begin{array}{l}\underline{\text { WBM }} \\
\text { Fekete et al. } \\
(2000)\end{array}$ & $\begin{array}{l}\underline{\text { WBMc }} \\
\text { Fekete et al. } \\
(2000)\end{array}$ & $\begin{array}{r}\frac{\text { WGHM}^{*}}{\text { Doll \& Fiedler }} \\
(2008)\end{array}$ & This Study \\
\hline Afghanistan & 55 & 55 & & 56 & 70 & 58 & 57 \\
\hline Albania & 27 & 27 & 19 & 23 & 23 & 19 & 12 \\
\hline Algeria & 11 & 14 & & 16 & 16 & 37 & 41 \\
\hline Angola & 148 & 184 & & 339 & 307 & 221 & 275 \\
\hline Argentina & 276 & 276 & 270 & 245 & 256 & 348 & 419 \\
\hline Armenia & 6.9 & 9.0 & 6.6 & 4.0 & 1.6 & 2.9 & 4.0 \\
\hline Australia & 492 & 492 & 352 & 266 & 291 & 774 & 758 \\
\hline Austria & 55 & 55 & & 57 & 43 & 52 & 52 \\
\hline Azerbaijan & 8 & 8.0 & 7.6 & 21 & 17 & 7.3 & 13 \\
\hline Bangladesh & 105 & 105 & & 143 & 131 & 108 & 181 \\
\hline Belarus & 37 & 37 & 35 & 50 & 35 & 29 & 58 \\
\hline Belgium & 12 & 12 & & 10 & 8.3 & 12 & 12 \\
\hline Belize & 16 & 16 & & 20 & 20 & 17 & 16 \\
\hline Benin & 10 & 10 & & 42 & 22 & 16 & 28 \\
\hline Bhutan & 78 & 95 & & 41 & 41 & 24 & 33 \\
\hline Bolivia & 304 & 304 & 361 & 260 & 324 & 357 & 311 \\
\hline Bosnia \& Herzegovina & 36 & 36 & & 42 & 41 & 31 & 22 \\
\hline Botswana & 2.4 & 3 & & 3.7 & 3.8 & -7.8 & 31 \\
\hline Brazil & 5,418 & 5,418 & 6,220 & 6,333 & 6,904 & 5,359 & 5,270 \\
\hline Brunei & 8.5 & & & 11 & 11 & 0.0 & 10 \\
\hline Bulgaria & 21 & 21 & & 15 & 21 & 22 & 22 \\
\hline Burkina Faso & 13 & 13 & & 47 & 14 & 13 & 47 \\
\hline Burundi & 10 & 4 & & 10 & 6.9 & 6.7 & 8.1 \\
\hline Cambodia & 121 & 121 & & 122 & 122 & 116 & 168 \\
\hline Cameroon & 273 & 273 & & 343 & 264 & 233 & 272 \\
\hline Canada & 2,850 & 2,850 & 3,287 & 2,114 & 2,555 & 2,702 & 2,751 \\
\hline
\end{tabular}




\begin{tabular}{|l|} 
Central African Republic \\
Chad \\
Chile \\
China \\
Colombia \\
Congo \\
Congo, DRC \\
Costa Rica \\
Cote d'Ivoire \\
Croatia \\
Cuba \\
Cyprus \\
Czech Republic \\
Denmark \\
Djibouti \\
Dominican Republic \\
Ecuador \\
Egypt \\
El Salvador \\
Equatorial Guinea \\
Eritrea \\
Estonia \\
Ethiopia \\
Falkland Is. \\
Fiji \\
Finland \\
France \\
French Guiana \\
Gabon \\
Gaza Strip \& West Bank \\
Georgia \\
\hline
\end{tabular}

\begin{tabular}{|c|c|}
\hline 141 & 141 \\
\hline 15 & 15 \\
\hline 884 & 884 \\
\hline 2,813 & 2,812 \\
\hline 2,112 & 2,112 \\
\hline 222 & 222 \\
\hline 900 & 900 \\
\hline 112 & 112 \\
\hline 77 & 77 \\
\hline 38 & 38 \\
\hline 38 & 38 \\
\hline \multicolumn{2}{|l|}{0.8} \\
\hline 13 & 13 \\
\hline 6.0 & 6 \\
\hline \multicolumn{2}{|l|}{0.3} \\
\hline 21 & 21 \\
\hline 432 & 432 \\
\hline 1.8 & 2 \\
\hline 18 & 18 \\
\hline 26 & 26 \\
\hline 2.8 & 3 \\
\hline 13 & 13 \\
\hline 122 & 110 \\
\hline \multicolumn{2}{|l|}{0.0} \\
\hline 29 & 29 \\
\hline 107 & 107 \\
\hline 179 & 179 \\
\hline \multicolumn{2}{|l|}{134} \\
\hline 164 & 164 \\
\hline \multicolumn{2}{|l|}{0.8} \\
\hline 58 & 58 \\
\hline
\end{tabular}

\begin{tabular}{|c|c|}
\hline & 970 \\
\hline 10 & 78 \\
\hline 354 & 379 \\
\hline 2,701 & 1,680 \\
\hline 1,200 & 1,402 \\
\hline & 174 \\
\hline 989 & 1,138 \\
\hline 110 & 99 \\
\hline & 101 \\
\hline & 28 \\
\hline 35 & 20 \\
\hline & 1.0 \\
\hline & 22 \\
\hline & 15 \\
\hline & 0.0 \\
\hline & 13 \\
\hline 265 & 210 \\
\hline & 0.0 \\
\hline 19 & 15 \\
\hline & 30 \\
\hline & 1.0 \\
\hline 12 & 20 \\
\hline & 310 \\
\hline & 29 \\
\hline & 104 \\
\hline 168 & 163 \\
\hline & 118 \\
\hline 205 & 188 \\
\hline 51 & 44 \\
\hline
\end{tabular}

\begin{tabular}{|c|c|c|}
\hline 120 & 120 & 225 \\
\hline 44 & 23 & 87 \\
\hline 376 & 381 & 198 \\
\hline 2,160 & 2,267 & 1,897 \\
\hline 1,668 & 1,836 & 1,354 \\
\hline 145 & 169 & 154 \\
\hline 847 & 955 & 786 \\
\hline 99 & 90 & 85 \\
\hline 84 & 92 & 126 \\
\hline 25 & 28 & 27 \\
\hline 20 & 31 & 38 \\
\hline 1.0 & 0.0 & 1.0 \\
\hline 15 & 16 & 20 \\
\hline 15 & 20 & 13 \\
\hline 0.0 & 0.7 & 0.5 \\
\hline 13 & 17 & 19 \\
\hline 126 & 368 & 212 \\
\hline 0.0 & -9.1 & 2.6 \\
\hline 15 & 12 & 13 \\
\hline 30 & 32 & 23 \\
\hline 0.7 & 7.4 & 5.4 \\
\hline 19 & 13 & 12 \\
\hline \multirow[t]{2}{*}{218} & 154 & 251 \\
\hline & 3.8 & 3.2 \\
\hline 29 & 21 & 14 \\
\hline 96 & 101 & 110 \\
\hline 172 & 227 & 190 \\
\hline 108 & 105 & 114 \\
\hline \multirow[t]{2}{*}{190} & 226 & 168 \\
\hline & & 1.0 \\
\hline 34 & 40 & 23 \\
\hline
\end{tabular}




\begin{tabular}{|l} 
Germany \\
Ghana \\
Greece \\
Greenland \\
Guatemala \\
Guinea \\
Guinea-Bissau \\
Guyana \\
Haiti \\
Honduras \\
Hungary \\
Iceland \\
India \\
Indonesia \\
Iran \\
Iraq \\
Ireland \\
Israel \\
Italy \\
Jamaica \\
Japan \\
Jordan \\
Kazakhstan \\
Kenya \\
Kuwait \\
Kyrgyzstan \\
Laos \\
Latvia \\
Lebanon \\
Lesotho \\
Liberia
\end{tabular}

$\begin{array}{rr}107 & 107 \\ 30 & 30 \\ 58 & 58 \\ 603 & \\ 109 & 109 \\ 226 & 226 \\ 16 & 16 \\ 241 & 241 \\ 13 & 13 \\ 96 & 96 \\ 6.0 & 6 \\ 170 & 170 \\ 1,280 & 1,261 \\ 2,838 & 2,838 \\ 129 & 129 \\ 35 & 35 \\ 49 & 49 \\ 0.8 & 1.0 \\ 183 & 183 \\ 9.4 & 9.0 \\ 430 & 430 \\ 0.7 & 1.0 \\ 75 & 75 \\ 21 & 20 \\ 0.0 & 0.0 \\ 49 & 47 \\ 190 & 190 \\ 17 & 17 \\ 4.8 & 5.0 \\ 5.2 & 200 \\ 200 & \\ & \\ 17\end{array}$

132
80
41
254
130
246
34
215
11
73
6.9
74
1,248
2,512
120
24
48
1.2
133
8.3
400
0.7
122
43

20
207
7.7
5.3
118

109
41
41
254
123
194
32
226
11
73
5.1
74
1,468
2,512
128
24
48
1.2
135
8.3
405
0.7
105
44

35
204
19
7.7
6.3
118

\begin{tabular}{|c|c|}
\hline 112 & 112 \\
\hline 34 & 64 \\
\hline 39 & 28 \\
\hline 306 & 60 \\
\hline 124 & 168 \\
\hline 156 & 205 \\
\hline 18 & 25 \\
\hline 182 & 220 \\
\hline 10 & 11 \\
\hline 83 & 92 \\
\hline 10 & 25 \\
\hline 119 & 68 \\
\hline 1,435 & 1,399 \\
\hline 2,292 & 2,163 \\
\hline 82 & 88 \\
\hline 5.7 & 21 \\
\hline 52 & 37 \\
\hline 3.2 & 1.3 \\
\hline 135 & 124 \\
\hline 6.6 & 9.5 \\
\hline 367 & 265 \\
\hline 3.1 & 2.0 \\
\hline 95 & 266 \\
\hline 50 & 73 \\
\hline 0.5 & 0.2 \\
\hline 21 & 23 \\
\hline 197 & 196 \\
\hline 18 & 17 \\
\hline 4.0 & 2.6 \\
\hline 4.0 & 5.0 \\
\hline 149 & 111 \\
\hline
\end{tabular}




\begin{tabular}{|l} 
Libya \\
Lithuania \\
Luxembourg \\
Macedonia \\
Madagascar \\
Malawi \\
Malaysia \\
Mali \\
Mauritania \\
Mexico \\
Moldova \\
Mongolia \\
Morocco \\
Mozambique \\
Myanmar \\
Namibia \\
Nepal \\
Netherlands \\
New Caledonia \\
New Zealand \\
Nicaragua \\
Niger \\
Nigeria \\
North Korea \\
Norway \\
Oman \\
Pakistan \\
Panama \\
Papua New Guinea \\
Paraguay \\
Peru \\
\end{tabular}

$\begin{array}{rr}0.6 & 1 \\ 16 & 16 \\ 1.0 & \\ 5.4 & 5.0 \\ 337 & 337 \\ 16 & 16 \\ 580 & 580 \\ 60 & 60 \\ 0.4 & 0 \\ 409 & 409 \\ 1.0 & 1.0 \\ 35 & 35 \\ 29 & 29 \\ 100 & 99 \\ 1,003 & 881 \\ 6.2 & 6 \\ 198 & 198 \\ 11 & 11 \\ 0.0 & \\ 327 & 327 \\ 190 & 190 \\ 3.5 & 4.0 \\ 221 & 221 \\ 67 & 67 \\ 382 & 382 \\ 1.4 & 1 \\ 55 & 52 \\ 147 & 147 \\ 801 & 801 \\ 94 & 916 \\ 1,616 & \end{array}$

14



40

345
1.2












143
2.3
275

0.8
17
0.6
4.3
359
58
539
78
0.8
303
1.1
17
11
183
736
0.6
125
7.4

250
140
4.1
337
55
224
1.0
26
110
723
64
785

$\begin{array}{rr}0.8 & 18 \\ 16 & 16 \\ 0.7 & 0.0 \\ 4.4 & 6.2 \\ 369 & 348 \\ 18 & -2.9 \\ 529 & 431 \\ 32 & 6.8 \\ 0.7 & 8.3 \\ 291 & 378 \\ 1.3 & 3.9 \\ 27 & 45 \\ 11 & 26 \\ 173 & 159 \\ 882 & 792 \\ 0.6 & 13 \\ 162 & 158 \\ 7.3 & 14 \\ & 7.7 \\ 250 & 283 \\ 140 & 152 \\ 2.8 & 50 \\ 345 & 278 \\ 55 & 55 \\ 233 & 289 \\ 1.0 & 13 \\ 27 & 55 \\ 110 & 81 \\ 723 & 72 \\ 63 & 306 \\ 531 & \end{array}$

13
22
0.7
6.1
319
39
482
94
13
359
8.1
94
34
206
736
26
98
13
5.7
258
155
29
354
49
214
2.7
44
78
589
109
691




\begin{tabular}{|l} 
Philippines \\
Poland \\
Portugal \\
Puerto Rico \\
Qatar \\
Romania \\
Russia \\
Rwanda \\
Saudi Arabia \\
Senegal \\
Serbia \& Montenegro \\
Sierra Leone \\
Slovakia \\
Slovenia \\
Solomon Is. \\
Somalia \\
South Africa \\
South Korea \\
Spain \\
Sri Lanka \\
Sudan \\
Suriname \\
Swaziland \\
Sweden \\
Switzerland \\
Syria \\
Tajikistan \\
Tanzania \\
Thailand \\
The Bahamas \\
The Gambia \\
\hline
\end{tabular}

479
54
38
7.1
0.1
42
4,313
10
2.4
26
44
160
13
19
45
6.0
45
65
111
53
30
88
2.6
171
40
7.1
66
84
225
0.0
3.0

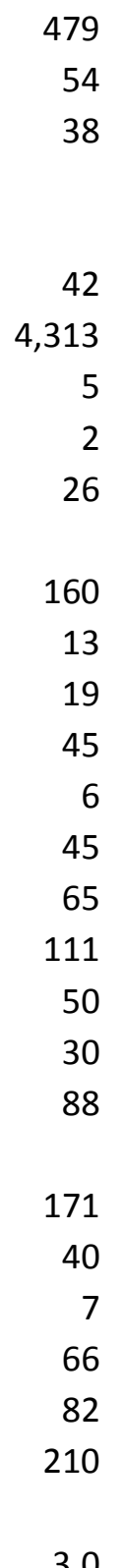

\begin{tabular}{|c|c|}
\hline & 343 \\
\hline & 58 \\
\hline \multirow[t]{4}{*}{19} & 35 \\
\hline & 6.7 \\
\hline & 0.0 \\
\hline & 38 \\
\hline \multirow[t]{3}{*}{4,053} & 2,909 \\
\hline & 5.8 \\
\hline & -0.1 \\
\hline \multirow[t]{8}{*}{21} & 37 \\
\hline & 120 \\
\hline & 17 \\
\hline & 21 \\
\hline & 26 \\
\hline & 0.2 \\
\hline & 39 \\
\hline & 55 \\
\hline \multirow[t]{2}{*}{109} & 83 \\
\hline & 52 \\
\hline \multirow[t]{6}{*}{35} & 139 \\
\hline & 138 \\
\hline & 1.8 \\
\hline & 108 \\
\hline & 50 \\
\hline & 8.1 \\
\hline \multirow[t]{4}{*}{47} & 5.6 \\
\hline & 187 \\
\hline & 268 \\
\hline & 0.0 \\
\hline 4.0 & 6.4 \\
\hline
\end{tabular}

\begin{tabular}{|c|c|c|}
\hline 343 & 278 & 251 \\
\hline 57 & 62 & 80 \\
\hline 35 & 41 & 30 \\
\hline 6.7 & 0.0 & 5.0 \\
\hline 0.0 & 0.1 & 0.0 \\
\hline 32 & 51 & 57 \\
\hline 3,704 & 3,550 & 4,251 \\
\hline 4.5 & 4.4 & 7.9 \\
\hline-0.1 & 25 & 6.0 \\
\hline \multirow[t]{2}{*}{25} & 8.8 & 51 \\
\hline & & 31 \\
\hline 120 & 103 & 113 \\
\hline 15 & 12 & 16 \\
\hline 20 & 14 & 15 \\
\hline 26 & 30 & 2 \\
\hline 0.2 & 13 & 18 \\
\hline 39 & 59 & 99 \\
\hline 60 & 51 & 47 \\
\hline 106 & 115 & 100 \\
\hline 52 & 39 & 47 \\
\hline 127 & 30 & 199 \\
\hline 130 & 102 & 162 \\
\hline 1.8 & 2.8 & 2.5 \\
\hline 125 & 177 & 151 \\
\hline 46 & 45 & 38 \\
\hline 8.1 & 11 & 17 \\
\hline 28 & 38 & 34 \\
\hline 125 & 129 & 246 \\
\hline 243 & 201 & 286 \\
\hline 0.0 & 2.4 & 1.6 \\
\hline 6.4 & 0.6 & 1.4 \\
\hline
\end{tabular}




\begin{tabular}{|c|c|c|c|c|c|c|c|}
\hline Timor-Leste & 0.0 & & & & & 4.0 & 3.9 \\
\hline Togo & 12 & 12 & & 25 & 11 & 11 & 17 \\
\hline Trinidad \& Tobago & 3.8 & 4.0 & & 2.1 & 2.1 & 0.0 & 1.9 \\
\hline Tunisia & 4.2 & 4 & & 5.5 & 5.5 & 8.1 & 8.6 \\
\hline Turkey & 227 & 227 & & 187 & 182 & 183 & 148 \\
\hline Turkmenistan & 1.4 & 1.0 & 1.1 & 4.7 & 4.7 & 7.9 & 13 \\
\hline Uganda & 39 & 39 & & 47 & 22 & 3.3 & 59 \\
\hline Ukraine & 53 & 53 & 51 & 71 & 55 & 70 & 152 \\
\hline United Arab Emirates & 0.2 & 0.0 & & 0.8 & 0.8 & 0.8 & 0.5 \\
\hline United Kingdom & 145 & 145 & & 126 & 126 & 177 & 152 \\
\hline United States & 2,818 & 2,818 & 2,930 & 2,208 & 2,297 & 2,382 & 2,254 \\
\hline Uruguay & 59 & 59 & 68 & 65 & 63 & 92 & 62 \\
\hline Uzbekistan & 16 & 16 & 10 & 11 & 12 & 14 & 25 \\
\hline Vanuatu & 0.0 & & & & & 11 & 4.3 \\
\hline Venezuela & 722 & 723 & & 852 & 765 & 773 & 728 \\
\hline Vietnam & 359 & 367 & & 263 & 265 & 235 & 269 \\
\hline Western Sahara & 0.0 & & & & & 2.1 & 0.4 \\
\hline Yemen & 2.1 & 4 & & 0.1 & 0.1 & 17 & 6.0 \\
\hline Zambia & 80 & 80 & & 275 & 254 & 71 & 202 \\
\hline Zimbabwe & 12 & 14 & & 35 & 40 & 31 & 53 \\
\hline
\end{tabular}

* The values of FAO, WRI, and WGHM are defined as renewable water resources. The remaining sources show the average annual runoff 
Table S2: Characteristics of the selected 29 world basins.

\begin{tabular}{|c|c|c|c|c|c|c|c|c|c|c|c|}
\hline \multirow[b]{2}{*}{$\begin{array}{l}\mathrm{N} \\
\mathrm{O}\end{array}$} & \multirow[b]{2}{*}{$\begin{array}{l}\text { River Basin } \\
\text { Name }\end{array}$} & \multicolumn{3}{|c|}{ SAGE* (2010) } & \multicolumn{4}{|c|}{ GRDC (1999) } & \multicolumn{3}{|c|}{ This Study } \\
\hline & & Station Name & Data Period & $\begin{array}{c}\text { Basin } \\
\text { Area } \\
{[\mathrm{km} 2]}\end{array}$ & $\begin{array}{c}\text { GRDC } \\
\text { station } \\
\text { number }\end{array}$ & Station Name & Data Period & $\begin{array}{c}\text { Basin Area } \\
{[\mathrm{km} 2]}\end{array}$ & $\begin{array}{l}\text { IWMI } \\
\text { Basin \# }\end{array}$ & Data Period & $\begin{array}{c}\text { Basin Area } \\
{[\mathrm{km} 2]}\end{array}$ \\
\hline 1 & Amazon & Obidos & $1928-1983$ & $4,618,746$ & 3629000 & Obidos - Porto & $1927-1998$ & $4,680,000$ & 1 & $1901-2002$ & $6,255,815$ \\
\hline 2 & Amur & Komsomolsk & 1933-1984 & $1,730,000$ & 2906901 & Bogorodskoye & 1963-1987 & $1,790,000$ & 3 & 1901-2002 & $1,655,920$ \\
\hline 3 & Brahmaputra & $\begin{array}{l}\text { Bahadurabad } \\
\text { Limite Internacional }\end{array}$ & 1969-1975 & 636,130 & 2651100 & $\begin{array}{l}\text { Bahadurabad } \\
\text { Lindero Internacional }\end{array}$ & 1969-1992 & 636,130 & 9 & $1901-2002$ & 635,779 \\
\hline 4 & Colorado & Norte & 1976-1979 & 631,960 & 4352100 & $\begin{array}{l}\text { Norte } \\
\text { Beaver Army Terminal }\end{array}$ & 1961-1995 & 631,960 & 23 & $1901-2002$ & 728,567 \\
\hline 5 & Columbia & The Dalles & 1960-1993 & 613,794 & 4115201 & Near Quincy & $1968-2010$ & 665,371 & 24 & $1901-2002$ & 685,718 \\
\hline 6 & Congo & Brazzaville & $1984-2002$ & $3,475,000$ & 1447150 & Brazzaville & $1971-1983$ & $3,475,000$ & 25 & $1901-2002$ & $3,082,792$ \\
\hline 7 & Danube & $\begin{array}{l}\text { Ceatallzmail } \\
\text { Dnieper Hydroelectric }\end{array}$ & 1921-1984 & 807,000 & 6742900 & $\begin{array}{l}\text { Ceatal Izmail } \\
\text { Kakhovskoye }\end{array}$ & $1921-2008$ & 807,000 & 27 & $1901-2002$ & $1,142,044$ \\
\hline 8 & Dnieper & Plant & $1952-1984$ & 463,000 & 6980802 & Vodokhranilishche Ges & $1959-1988$ & 482,000 & 28 & $1901-2002$ & 767,324 \\
\hline 9 & $\begin{array}{l}\text { Ganges } \\
\text { Huang He }\end{array}$ & Paksay & 1965-1975 & 846,900 & 2646100 & Paksey & 1969-1975 & 846,900 & 34 & $1901-2002$ & $1,032,334$ \\
\hline 10 & (Yellow River) & Sanmenxia & 1976-1979 & 688,421 & 2180800 & Huayuankou & $1946-2004$ & 730,036 & 41 & $1901-2002$ & $1,047,855$ \\
\hline 11 & Indus & Kotri & 1973-1979 & 832,418 & 2335950 & $\begin{array}{l}\text { Kotri } \\
\text { Phnom Penh (Chroui }\end{array}$ & 1936-1979 & 832,418 & 45 & $1901-2002$ & $1,092,691$ \\
\hline 12 & Mekong & Mukdahan & 1924-1987 & 391,000 & 2569002 & Changvar) & $1960-1973$ & 663,000 & 60 & 1901-2002 & 826,867 \\
\hline 13 & Mississippi & Vicksburg, Missisippi & 1965-1983 & $2,964,254$ & 4127800 & Vicksburg, Ms & $1928-2009$ & $2,964,255$ & 62 & $1901-2002$ & $3,144,274$ \\
\hline 14 & Murray & Lock9 Upper & 1965-1984 & 991,000 & 5204268 & Lock 9 Upstream & $1965-1984$ & 991,000 & 64 & $1901-2002$ & $1,032,479$ \\
\hline 15 & Niger & Gaya & $1952-1990$ & $1,000,000$ & 1834101 & Lokoja & $1970-2006$ & --- & 66 & $1901-2002$ & $2,229,504$ \\
\hline 16 & Nile & AswanDam & 1869-1984 & --- & 1362600 & Aswan Dam & $1869-1984$ & --- & 67 & $1901-2002$ & $2,788,360$ \\
\hline 17 & $\mathrm{Ob}$ & Salekhard & 1930-1984 & $2,430,000$ & 2912600 & Salekhard & $1930-2003$ & $2,949,998$ & 75 & $1901-2002$ & $2,790,218$ \\
\hline 18 & Orange & Vioolsdrif & 1964-1986 & 850,530 & 1159100 & Vioolsdrif & $1935-2001$ & 850,530 & 78 & $1901-2002$ & 613,453 \\
\hline 19 & Orinoco & Musinacio & 1973-1975 & --- & 3206720 & Puente Angostura & 1923-1989 & 836,000 & 79 & $1901-2002$ & $1,203,565$ \\
\hline 20 & Parana & Corrientes & 1969-1979 & $2,300,000$ & 3618001 & Careiro & $1977-2010$ & $2,583,079$ & 81 & $1901-2002$ & $2,659,381$ \\
\hline 21 & Rhine & Rees & 1936-1984 & 159,680 & 6435060 & Lobith & 1901-2007 & 160,800 & 85 & $1901-2002$ & 220,414 \\
\hline 22 & Rio Grande & Laredo,Texas & $1900-1972$ & 352,178 & 4351900 & Matamoros & $1934-2000$ & 450,902 & 88 & $1901-2002$ & 690,266 \\
\hline 23 & Syr Darya & Tyumen-Aryk & 1930-1984 & 219,000 & 2916200 & Tyumen-Aryk & 1930-1986 & 219,000 & 104 & $1901-2002$ & 465,312 \\
\hline & Euphrates & D.S.Hindiya B. & 1968-1972 & 274,100 & 2595400 & Hindiya & 1923-1972 & 274,100 & --- & --- & --- \\
\hline 24 & Tigris & Baghdad & 1968-1972 & 134,000 & 2595700 & Baghdad & 1906-1972 & 134,000 & --- & --- & --- \\
\hline
\end{tabular}




\begin{tabular}{|c|c|c|c|c|c|c|c|c|c|c|c|}
\hline & $\begin{array}{l}\text { Tigris- } \\
\text { Euphrates }\end{array}$ & --- & --- & 408,100 & --- & --- & --- & 408,100 & 107 & 1901-2002 & 841,321 \\
\hline 25 & Ural & $\begin{array}{l}\text { Kushum } \\
\text { Volgograd }\end{array}$ & 1915-1984 & 190,000 & 2919200 & Kushum & $1915-1988$ & 190,000 & 111 & 1901-2002 & 922,085 \\
\hline 26 & Volga & Hydroelectric Plant & $1879-1984$ & $1,360,000$ & 6977100 & Volgograd Power Plant & $1879-2002$ & $1,360,000$ & 114 & $1901-2002$ & $1,603,982$ \\
\hline 27 & Yenisei & Igarka & 1936-1984 & $2,440,000$ & 2909150 & Igarka & $1936-2003$ & $2,440,000$ & 120 & $1901-2002$ & $2,566,163$ \\
\hline 28 & $\begin{array}{l}\text { Zambezi } \\
\text { Zhu Jiang (Xi }\end{array}$ & Matundo- Cais & 1976-1979 & 940,000 & 1891500 & Matundo-Cais & $1976-1979$ & 940,000 & 123 & $1901-2002$ & $2,007,908$ \\
\hline 29 & Jiang) & Wuzhou3 & $1976-1983$ & 329,705 & 2186800 & Wuzhou 3 & $1915-2004$ & 329,705 & 124 & 1901-2002 & 682,308 \\
\hline
\end{tabular}

* SAGE: contains a compilation of monthly mean river discharge data for over 3500 sites worldwide from various data sources (RivDis2.0, the United States Geological Survey, Brazilian National

Department of Water and Electrical Energy, and HYDAT-Environment Canada); the period of record for each station is variable, from 3 years to greater than 100. 
Table S3: Average annual runoff $\left[\mathrm{km}^{3} / \mathrm{yr}\right]$ at the basin scale in comparison with other data-based and model based estimates.

\begin{tabular}{|c|c|c|c|c|c|c|c|c|c|c|c|}
\hline \multirow[b]{2}{*}{ No. } & \multirow[b]{2}{*}{ River Basin Name } & \multicolumn{2}{|c|}{ Observed Data } & \multicolumn{3}{|c|}{ Data-Based } & \multicolumn{5}{|c|}{ Model-Based } \\
\hline & & $\begin{array}{l}\text { SAGE } \\
(2010)\end{array}$ & $\begin{array}{l}\text { GRDC } \\
(1999)\end{array}$ & $\begin{array}{c}\text { Shiklomanov } \\
(2000)\end{array}$ & $\begin{array}{l}\text { WRI } \\
(2000)\end{array}$ & $\begin{array}{c}\text { Probst \& Tardy } \\
(1987)\end{array}$ & $\begin{array}{c}\frac{\text { PCR-GLOBWB }}{\text { Weiland et al. }} \\
\text { (2010) }\end{array}$ & $\begin{array}{c}\frac{\text { WaterGAP } 2}{\text { Alcamo et al. }} \\
\text { (2003) }\end{array}$ & $\begin{array}{l}\frac{\text { WBM }}{\text { Fekete et al. }} \\
(2000)\end{array}$ & $\begin{array}{l}\frac{\text { WBMc }}{\text { Fekete et al. }} \\
(2000)\end{array}$ & This Study \\
\hline 1 & Amazon & 5,326 & 4,902 & 6,923 & 6,729 & 4,729 & 5,992 & 5,436 & 5,658 & 6,813 & 4,928 \\
\hline 2 & Amur & 323 & 307 & 360 & 323 & --- & --- & --- & 334 & 402 & 361 \\
\hline 3 & Brahmaputra & 690 & 620 & -- & -- & -- & 1,519 & --- & 651 & 861 & 587 \\
\hline 4 & Colorado & 4 & 2 & --- & 15 & --- & --- & --- & 13 & 17 & 58 \\
\hline 5 & Columbia & 205 & 172 & 236 & 234 & -- & -- & -- & 147 & 226 & 197 \\
\hline 6 & Congo & 1,297 & 1,264 & 1,320 & 1,273 & -- & 1,318 & -- & 1,484 & 1,087 & 1,074 \\
\hline 7 & Danube & 203 & 205 & 225 & 204 & 172 & 202 & 185 & 370 & 333 & 365 \\
\hline 8 & Dnieper & 43 & 47 & 53 & 51 & -- & -- & --- & 119 & 84 & 202 \\
\hline 9 & Ganges & 381 & 351 & 1,386 & --- & --- & 379 & 357 & 391 & 427 & 471 \\
\hline 10 & Huang He (Yellow River) & 44 & 38 & -- & 54 & -- & -- & -- & 76 & 93 & 139 \\
\hline 11 & Indus & 90 & 76 & 226 & 146 & --- & 208 & --- & 172 & 151 & 98 \\
\hline 12 & Mekong & 410 & 252 & 505 & 511 & 262 & 505 & 233 & 473 & 458 & 533 \\
\hline 13 & Mississippi & 543 & 537 & 510 & 632 & --- & 402 & 442 & 652 & 626 & 732 \\
\hline 14 & Murray & 8 & 8 & 24 & 24 & -- & 24 & -- & 15 & 15 & 109 \\
\hline 15 & Niger & 158 & 36 & 302 & 295 & --- & 189 & -- & 521 & 456 & 549 \\
\hline 16 & Nile & 87 & 87 & 161 & 316 & -- & -- & 36 & 355 & 249 & 343 \\
\hline 17 & $\mathrm{Ob}$ & 400 & 393 & 404 & 444 & --- & -- & -- & 392 & 392 & 714 \\
\hline 18 & Orange & 9 & 5 & -- & 11 & -- & 12 & -- & 9 & 9 & 30 \\
\hline 19 & Orinoco & 980 & 906 & 1,007 & 1,467 & -- & -- & -- & 1,281 & 1,152 & 1,171 \\
\hline 20 & Parana & 391 & 546 & -- & 560 & --- & 568 & -- & 726 & 561 & 788 \\
\hline 21 & Rhine & 70 & 72 & -- & -- & -- & 69 & -- & 103 & 93 & 99 \\
\hline 22 & Rio Grande & 1 & 4 & -- & 7 & -- & -- & -- & 5 & 4 & 36 \\
\hline 23 & Syr Darya & 17 & 17 & --- & 26 & --- & -- & --- & 28 & 38 & 44 \\
\hline 24 & Tigris-Euphrates* & 57 & 50 & --- & 96 & --- & --- & --- & 113 & 108 & 93 \\
\hline 25 & Ural & 9 & 9 & -- & 7 & -- & -- & -- & 21 & 17 & 83 \\
\hline 26 & Volga & 257 & 255 & 255 & 252 & 257 & 254 & 240 & 309 & 296 & 425 \\
\hline
\end{tabular}




\begin{tabular}{|c|c|c|c|c|c|c|c|c|c|c|c|}
\hline 27 & Yenisey & 583 & 558 & 618 & 606 & --- & -- & --- & 458 & 733 & 757 \\
\hline 28 & Zambezi & 105 & 105 & 154 & -- & --- & 107 & --- & 532 & 453 & 456 \\
\hline 29 & Zhu Jiang (Xi Jiang) & 217 & 223 & -- & -- & --- & -- & 165 & 374 & 464 & 420 \\
\hline
\end{tabular}

*For RivSim and GRDC, Euphrates and Tigris runoff values are, 19, 18, 38, and 32, respectively. 